\title{
The best mood environment design for Shang-pool Arcadia
}

\author{
${ }^{1, \mathrm{a}}$ Fei Jiang ${ }^{2, \mathrm{~b}}$ Peter Appleton ${ }^{3, \mathrm{c}}$ Yingjie Yu \\ 1,3 Shanghai University, Shanghai, 200444 ,China \\ ${ }^{2}$ Liverpool John Moores University, United Kingdom \\ A,b pcboy0309@sina.com
}

Keywords: Arcadia; Interactive; Liverpool; Shanghai, Canoe; Navigation; Wellbeing

\begin{abstract}
This is an ongoing research project between Art and Science faculties of Liverpool John Moores and Shanghai Universities. The collaboration centers around the creation of interactive and telematics non-verbal situations of meeting and cooperation. This suited the developing relationship between collaborating staff and $\mathrm{PhD}$ students in China and the $\mathrm{UK}$, bringing together research interests around a common technical objective, and the exploration and assimilation of cultural differences and empathies.
\end{abstract}

\section{Introduction}

Throughout the development history of virtual art, there is one theme to be reckoned with:utilization and interpretation of space. Virtual art has expanded human perception capability via technologies such as network, virtual reality and mixed reality, breaking originally inherent barrier of material space and letting people enter one space not limited by space-time and unimpeded by culture. It is also the inevitable result of continuous exploration of mankind to space. The development of virtual art reflects the process that artists continuously discover, perceive and expand space via latest technology at that time. Compounded by that the creation carrier of virtual art includes material space and digital space, spatial design on virtual art has become the core contents of its artistic creation. After development in recent years, innovation of information technology has brought more possibilities for spatial design on virtual art. How shall artists combine new technology to conduct creation on virtual art in future has become one topic to be resolved.

As one virtual art project, Shang-pool Acadia has been divided into three sub-headings: Picnic in Arcadia, Voyages in Arcadia and Gazing into Arcadia. The subject of Arcadia was inspired by a visit to Hangzhou with its idealised and engineered landscape configured by the Tongzhi Emperor and now adjoining a city of nine million people. We drew on the traditions and perceptions of nature and its relationship to the city in both Chinese and British cultures. It explores the notion of the idyll and nature as a place of recreation, contemplation, nourishment, and of meeting.

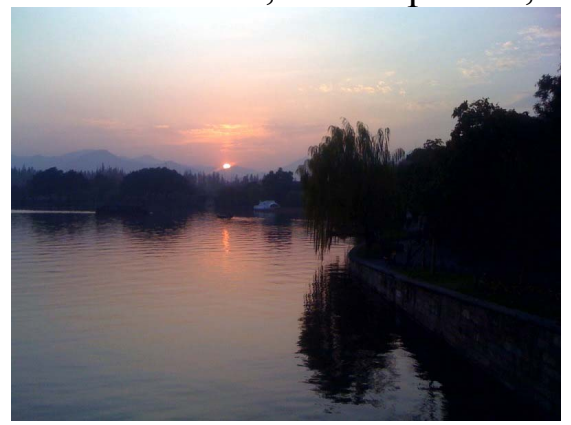

Figure 1: Hangzhou Great Lake Source: Appleton 2010Source:

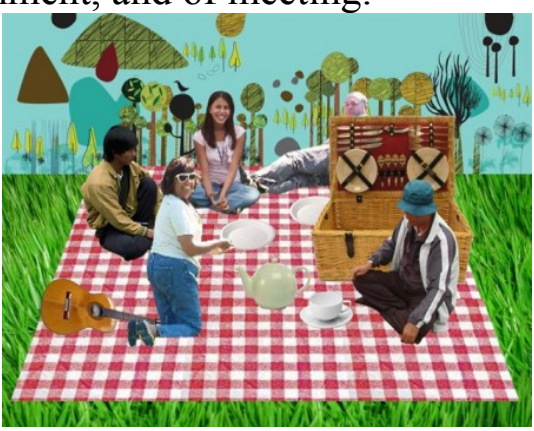

Figure 2: Picnic in Arcadia

Sermon 2011

Like all technological applications for mass consumers, as Virtual Reality applications became more widespread, these systems also became increasingly affordable."The hopes of the pioneer VR researchers of the mid-1980's are beginning to be realized as hardware and software development 


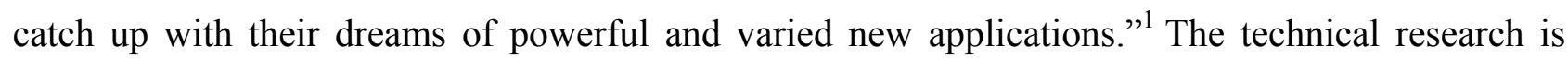
centered on the convergence of virtual, augmented, interactive, telematic and telepresence technologies in the creation of mixed reality shared experiences. Telepresence is defined as follows:"When a VR system succeeds in creating the interactive feedback loop between our perceptions and the real environment, then we have full telepresence. The term "telepresence" comes from the Greek word tele, meaning remote, and presence, a complex notion that we understand intuitively but that takes effort to unpack." 2 The virtual worlds of Shang-pool Arcadia become places of meeting, exploration and imagining, but, uniquely, they were also projected within real places at a series of events in parks and public spaces in Liverpool and Shanghai between 2010 and 2013, blurring the boundaries between real and virtual experience. This project entailed the development of novel forms of interaction with these virtual worlds. A virtual park has been built in Second Life, modelled on Stanley Park in Liverpool, which forms the starting point for the creation of a hybrid Anglo-Sino Arcadia. This elaborate model of Stanley Park in North Liverpool, painstakingly constructed in Second Life included many authentic details such as pagodas, lakes and bridges. We worked with a range of geographical data which was surfaced and textured from photographs taken in the park. The model of Stanley Park became the setting for a virtual meeting place, enabling people from Liverpool and Shanghai to mingle, talk and play together amid the imagined landscapes of Second Life. This park had been chosen because of its series of connected lakes and waterways later enabling the use of a canoe as a means of navigation and exploration.

\section{Picnic in Arcadia}

At the first Shang-pool Arcadia event, Picnic in Arcadia, public audiences at the Bluecoat in Liverpool and researchers at Shanghai University simultaneously met in a virtual picnic space. Shang-pool Arcadia builds upon previous collaborative research undertaken with research colleague Professor Paul Sermon at the University of Salford investigating presence and mixed realities. The picnic seemed an appropriate first social event with its resonance of sharing food in an outdoor setting.

"The essential attribute of interactive art is "interaction". It is a kind of art that makes connections between people and themselves, people and people as well as people and the environment."3Utilising the latest blue screen and HD videoconferencing technology the installation brought public participants together within a shared telepresent urban picnic scene. Merging live camera views of remote audiences together and placing them within a simulated arcadia environment, together with computer animated elements that were triggered and controlled by the audience through a unique motion tracking interface integrated within the installation. When a member of the audience discovers their image on screen they immediately enter the telepresent space, watching a live image of themselves sitting on a picnic blanket next to another person. They soon start to explore the space and understand they are now in complete physical control of a telepresent body that can interact with another person in an illustrated enchanted arcadian scene, complete with animated characters that respond to their movement and actions. This work, allowed the public to engage and interact while simply passing through or relaxing having lunch. This playful environment merges two public spaces together, creating a third otherworldly space on screen where people can interact with others across the globe on a virtual picnic blanket, allowing the audience to explore alternative networked spaces.

\section{Voyages in Arcadia}

At the second event audiences navigated the waterways of this virtual world seated in a Canadian canoe using Wii-like paddles whilst sharing a collective viewpoint of the virtual park projected on to a screen in front of them. The canoe had been chosen because of its association with new worlds and virgin wilderness. Fixed indoor canoes situated in Shanghai and Liverpool were controllers for the

\footnotetext{
1 Tony Feldman, Virtual Reality 91: Impacts and Applications (London: Meckler Ltd., 1991).

${ }^{2}$ Michael Heim, Virtual Realism (New York: Oxford University Press, 1998), p. 12.

${ }^{3}$ Paul Sermon, 2012 Interactive Design App Workshop(Shanghai: Shanghai University Press,2013), p.9.
} 
virtual canoes that sailed on the lake of the virtual Arcadia. The action of the paddles by the people in the fixed canoe caused reciprocal motion and navigation of the virtual canoe in second life. The co-operation of those paddling was essential as each paddle stroke on either side affected the motion and direction of the boat. The collective relative viewing position allowed a shared vista and experience of the projected voyage. ${ }^{4}$
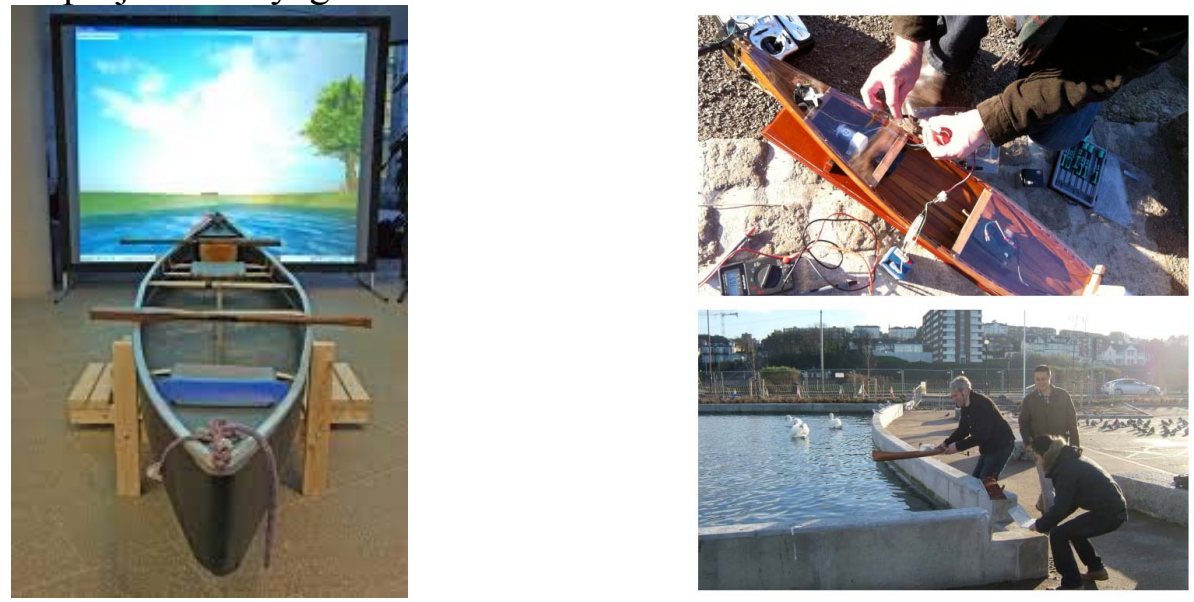

Figure 3: Navigating Canoe with Paddles Figure 4: Sailing a Model Canoe on the Actual Waterways Source: Appleton 2012

Source: Jiang 2012

"In general there is no relationship between beauty and control. Supported by the technique of automation and computer science, it is possible to use entities showing rational visual motion by precise control. This is the pursuit of aesthetic ideology of the works." ${ }^{, 5} \mathrm{We}$ also sought to add another layer of reality by sailing a model canoe on the actual waterways and lakes of Stanley Park, relaying a live video feed to the participating audiences in Liverpool and Shanghai. The ambition was to overlay and merge the actual viewpoint from the drone canoe with its virtual viewpoint in the Stanley Park model. We called the remotely controlled model the Arcadian Drone Canoe. This was a benign parody of the drone aircraft used to remotely bomb distant targets. Using the same controlling data from the fixed canoes, Arduino technologies received this through Wi-Fi, allowing the canoe's motion and direction to correspond to the actions of the Wii-like paddles at a distance. If everyone paddles on the left the boat will turn right, while more paddles produce more speed, and so on. A live video image from the prow of the canoe was Skyped back to the screens in front of the fixed canoes. We now had a second layer of reality, the live viewpoint from the drone. ${ }^{6}$

At another Shang-Pool event, Blossom Time, featuring two virtual canoes, one paddled from the fixed canoe in Shanghai and one paddled from a fixed canoe in Liverpool. These met on the virtual lake of Stanley Park. This experience of cooperative journeying has become an area of further research. We are developing ways of making paddle control more fluent using the Kinect system and also exploring the more immersive possibilities of the new generation of 3-D glasses. ${ }^{7}$

As control and media technologies become further developed we are able to extend the possibilities of shared experience and begin to explore softer goals of presence and wellbeing.

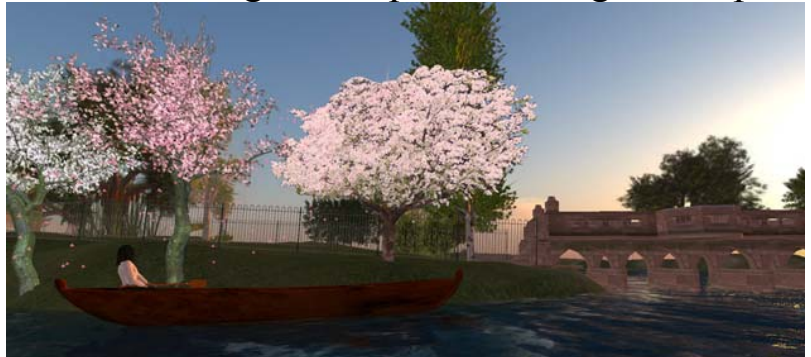

Figure 5: Blossom Time Source: Appleton 2013

\footnotetext{
${ }^{4} \mathrm{~A}$ video of an event testing the canoe navigation can be found here: http://www.youtube.com/watch? $\mathrm{v}=6 \mathrm{OEZdNNIdbM}$.

${ }^{5}$ Wang Zheng, 2012 Interactive Design App Workshop(Shanghai: Shanghai University Press,2013), p.12.

${ }^{6} \mathrm{~A}$ video of the testing of the drone canoe can be found at: http://www.youtube.com/watch?v=R9utbJaHre4.

${ }^{7}$ A video of the testing of the Kinetic Paddle control can be found at: http://www.youtube.com/watch? $v=x J Z 4 X J 8 n J q E$.
} 


\section{Gazing into Arcadia}

At the third Shang-pool Arcadia event, the concrete exhibition form of Gazing into Arcadia is: when audiences observe site via telescope in work, screen would show that site environment is overlaid with a layer of virtual images, and these images show cultural elements of Shanghai and Liverpool. Photographed photos would be displayed on nearby multi-touch interactive device for checking, amending or uploading to social network by site audiences, thereby forming network interactivity. Such work mainly combines relevant technologies of mixed reality, network interactivity and mobile platform, which can not only attract site audiences to consciously participate in interaction, but also feed back interaction contents to internet in real-time. Meanwhile, it can let on-line and off-line audiences form interaction. "Social network websites offer an unprecedented opportunity to understand the impact of a person's structural position on everything from their tastes to their moods to their health." ${ }^{8}$ Such networked interaction mode can strengthen degree of participation of the public, making "Gazing into Arcadia" become one virtual art work with public nature.

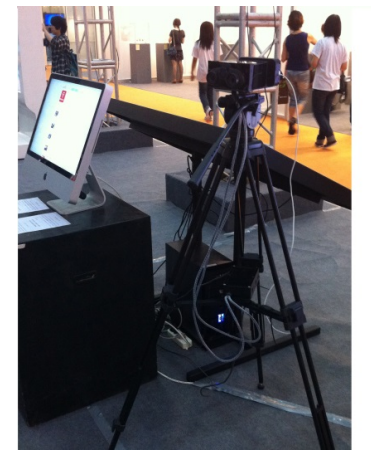

Figure 6: Gazing into Arcadia

Source: Jiang 2013

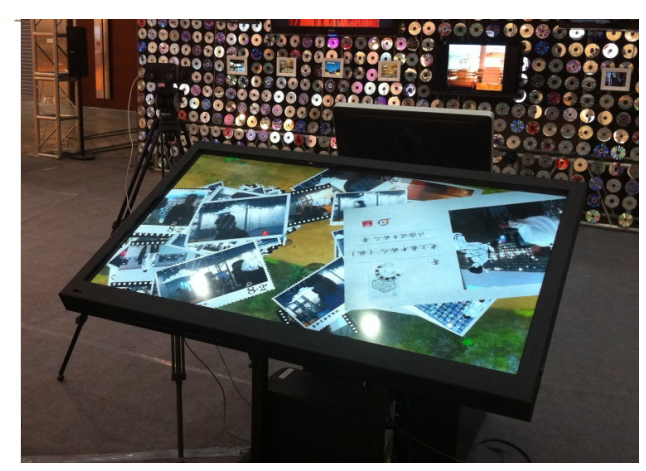

Figure 7: Image contents photographed by audiences Source: Jiang 2013

As per interaction mode, such work can be divided into three parts: 1. site material gathering; 2 . local material amending; 3 . social network interactivity. The whole set of interaction system is mainly composed by image synthesis equipment, multi-touch interaction device, and network communication equipment. At exhibition site, there are three interactive devices for interaction of audiences: 1. 55inch multi-touch interactive device; 2. telescope with holder; 3. computer. Multi-touch screen shows frames gathered by site audiences via telescope, while computer shows comment information of online audiences.

Ivan Edward Sutherland created the first computer-aided HMD in 1968, with internal sensors that tracked the user's head movements, and event Oliver Grau has subsequently called "the first step on the way to a media utopia."9 Like HMD, the telescope is one video input equipment; and hardware device is mainly composed by iPod Touch and Arduino module. Taking telescope as shell is just one metaphor design on interactive media, enabling audiences to intuitively understand how to operate it. Moreover, screen of iPod Touch is intentionally done with cover simulating telescope lens. Thus, when audiences see iPod Touch screen from two lens hole of telescope, they would mistakenly believe that it is content seen from telescope lens. Multi-touch screen provides checking, amending and uploading pictures for site audiences. Frames gathered by telescope would show on screen, and audiences can upload it to social network after selecting pictures from touch screen.

Image contents photographed by audiences via telescope would be transmitted to multi-touch interactive device via wireless router. The host of such device is installed with $3 \mathrm{G}$ network card, by virtue of which uploading contents can be distributed to internet. When audiences observe site via telescope, they can freely change angles, freely allocate the combination between site environment and virtual image on screen, and conduct photographing and uploading. Photographed photos would be displayed on nearby multi-touch interactive device. Site audiences can check, amend or upload it

\footnotetext{
8 Lewis K, Kaufman J, Gonzalez M, Wimmer A, Christakis N. Tastes, Ties, and Time: A New Social Network Dataset Using Facebook.com. (Social Networks, Volume 30, Issue 4 2008), P.330.

${ }^{9}$ Oliver Grau, Virtual Art: From Illusion to Immersion(MIT Press, 2004), P.163.
} 
to social network via touch mode. Net friends on social network can comment on picture contents, and comment contents would be displayed on computer screen for checking and reviewing of site audiences, thereby forming on-line and off-line simultaneous interaction.

Seen from exhibition results of this work, it has basically achieved expected target, attracting lots of site audiences and network audiences to participate in interactive space of work. It can be seen from some sampled network comments that lots of audiences are very interested in the representation form of virtuality and reality combination. Some parodying pictures of site audiences were repeatedly re-posted. Therefore, fans quantity of twitter account registered via work substantially increased on the very day of exhibition.

\section{Acknowledgement}

The authors would like to think the research and production team at Liverpool John Moores University: Andy Laws for creation of 3-D environments, Dr David Lamb for design and implementation of internet control and communication systems, Dr Chris Carter for ongoing development of kinetic paddle control system. At the Shanghai University: Dr Zheng Wang for implementation of communication systems.

\section{REFERENCES}

Feldman, Tony. 1991. Virtual Reality 91: Impacts and Applications. London: Meckler Ltd.,.

Grau, Oliver. 2004. Virtual Art: From Illusion to Immersion. MIT Press: p.163.

Heim, Michael. 1998. Virtual Realism . New York: Oxford University Press. p. 12.

Lewis K, Kaufman J, Gonzalez M, Wimmer A, Christakis N. 2008. "Tastes, Ties, and Time: A New Social Network Dataset Using Facebook.com.” Social Networks. Volume 30: p.330.

Sermon, Paul. 2013. "Telematic Art.” 2012 Interactive Design App Workshop: p.9.

Zheng, Wang. 2013. “Art of Control.” 2012 Interactive Design App Workshop: p.12. 